\title{
Density-Functional Theory of Surface Diffusion and Epitaxial Growth of Metals
}

\author{
C. Ratsch, P. Ruggerone, and M. Scheffler \\ Fritz-Haber-Institut der Max-Planck-Gesellschaft \\ Faradayweg 4-6, D-14195 Berlin-Dahlem, Germany
}

This paper gives a summary of basic concepts of density-functional theory (DFT) and its use in state-of-the-art computations of complex processes in condensed matter physics and materials science. In particular we discuss how microscopic growth parameters can be determined by DFT and how on this basis macroscopic phenomena can be described. To reach the time and length scales of realistic growth conditions, DFT results are complemented by kinetic Monte Carlo simulations.

\section{INTRODUCTION}

The microscopic processes governing epitaxial growth typically are rather complex since they involve the making and breaking of chemical bonds as well as the dynamics of the nuclei. With recent progress in the developments of new methods and techniques and the availability of faster computers, density-functional theory (DFT) calculations have evolved into a powerful tool to study growth phenomena (as well as other complex processes in condensed matter physics, materials science, and chemistry). The rate of a microscopic process $j$ that occurs during growth, such as diffusion, usually has the form $\Gamma^{(j)}=\Gamma_{0}^{(j)} \exp \left(-E_{\mathrm{d}}^{(j)} / k_{\mathrm{B}} T\right)$, where $\Gamma_{0}^{(j)}$ is the effective attempt frequency, $T$ the temperature, $k_{\mathrm{B}}$ the Boltzmann constant, and $E_{\mathrm{d}}^{(j)}$ is the energy barrier that needs to be overcome for the event $j$ to take place. This equation reflects the idea that an adatom experiences many stable and metastable sites at the surface, and that the diffusive motion brings it from one minimum to an adjacent one on the free energy surface in the space of the reaction coordinates. The effective attempt frequency $\Gamma_{0}^{(j)}$ contains the terms due to the adatom and substrate vibrations (see. Ref. 1 for more details).

It is at the heart of theoretical studies of surface diffusion and growth phenomena to calculate the ground-state total energy of the adsorbate system for a dense mesh of adatom positions. This yields the so-called potential-energy surface (PES) which is the 
potential energy experienced by the diffusing adatom,

$$
E^{\mathrm{PES}}\left(X_{\mathrm{ad}}, Y_{\mathrm{ad}}\right)=\min _{Z_{\mathrm{ad}},\left\{\mathbf{R}_{I}\right\}} E^{\mathrm{tot}}\left(X_{\mathrm{ad}}, Y_{\mathrm{ad}}, Z_{\mathrm{ad}},\left\{\mathbf{R}_{I}\right\}\right)
$$

where $E^{\text {tot }}\left(X_{\mathrm{ad}}, Y_{\mathrm{ad}}, Z_{\mathrm{ad}},\left\{\mathbf{R}_{I}\right\}\right)$ is the ground-state energy of the many-electron system (also referred as the total energy) at the atomic configuration $\left(X_{\mathrm{ad}}, Y_{\mathrm{ad}}, Z_{\mathrm{ad}},\left\{\mathbf{R}_{I}\right\}\right)$. According to Eq. (1) the PES is the minimum of the total energy with respect to the $z$-coordinate of the adatom and all coordinates of the substrate atoms $\left\{\mathbf{R}_{I}\right\}$. Assuming that vibrational effects can be neglected, the minima of the PES represent stable and metastable sites of the adatom. Note, this PES refers to slow motion of nuclei and assumes that for any atomic configuration the electrons are in their respective ground state. Thus, it is assumed that the dynamics of the electrons and of the nuclei are decoupled. This is the Born-Oppenheimer approximation which for not too high temperatures is usually well justified.

Now consider all possible paths $j$ to get from one stable or metastable adsorption site, $\mathbf{R}_{\mathrm{ad}}$, to an adjacent one, $\mathbf{R}_{\mathrm{ad}}{ }^{\prime}$. The energy difference $E_{\mathrm{d}}^{j}$ between the energy at the saddle point along $j$ and the energy of the start geometry is the barrier for this particular path. The diffusion barrier then is the minimum value of all $E_{\mathrm{d}}^{j}$ of all possible paths which connect $\mathbf{R}_{\mathrm{ad}}$ and $\mathbf{R}_{\mathrm{ad}}{ }^{\prime}$, and the lowest energy saddle point is called the transition state. We note this definition strictly applies only to cases where the vibrational energy is negligible, which is typically justified when the diffusion barrier is not too small, and the temperature is not too high. We also note that for non zero temperatures, i.e., when vibrations are thermally excited, vibrational entropy needs to be considered, which will enter the attempt frequency of the hopping rate. Although often only the path with the most favorable energy barrier is important, it may happen that several paths exist with comparable barriers or that the PES consists of more than one sheet (e.g., Ref. 2). Then the effective barrier measured in an experiment (or a molecular dynamics simulation) represents a proper average over all possible pathways. The above description obviously applies to simple jumps of an adatom (i.e., diffusion by hopping), but it also holds for the diffusion by atomic exchange where the diffusing adatom displaces a substrate atom. ${ }^{3-7}$

Aiming at a calculation and understanding of the PES of a diffusing atom, it is obvious that the interplay between the breaking and making of chemical bonds and the atomic relaxations needs to be accounted for by an accurate, quantum-mechanical description of the many-electron system. This can be achieved by modern densityfunctional theory calculations that combine electronic self-consistency and efficient geometry optimization (see for example Ref. 8-10 and references therein).

In the following two sections we describe briefly the basic concepts of densityfunctional theory (DFT) and of its application to surface problems. We then discuss some examples where DFT has been used to calculate growth parameters and compare the results with experiments. Finally, an example is given that shows how DFT calculations can be used in combination with kinetic Monte Carlo simulations to describe and analyze epitaxial growth.

\section{DENSITY-FUNCTIONAL THEORY: BASIC CONCEPTS}

The total energy of an $N$-electron, poly-atomic system is given by the expectation value of the many-particle Hamiltonian using the many-body wave-function of the 
electronic ground state. For a solid or a surface the calculation of such an expectation value is impossible when using a wave-function approach. However, as has been shown by Hohenberg and Kohn, ${ }^{11}$ the ground-state total energy can also be obtained without explicit knowledge of the many-electron wave-function, but by minimizing an energy functional $E[n]$. This is the essence of density-functional theory (DFT), which is primarily (though in principle not exclusively) a theory of the electronic ground state, couched in terms of the electron density $n(\mathbf{r})$ instead of the many-electron wave-function $\Psi\left(\left\{\mathbf{r}_{i}\right\}\right)$.

The important theorem of Hohenberg and Kohn ${ }^{11}$ (see also Levy ${ }^{12}$ ) tells: The specification of a ground state density $n(\mathbf{r})$ determines the corresponding external potential $v^{\text {ext }}(\mathbf{r})$ uniquely (to within an additive constant),

$$
n(\mathbf{r}) \rightarrow v^{\mathrm{ext}}(\mathbf{r})
$$

The external potential $v^{\text {ext }}(\mathbf{r})$ is typically (and definitely for our purpose here) the Coulomb potential due to the nuclei. While the other direction $\left[v^{\operatorname{ext}}(\mathbf{r}) \rightarrow n(\mathbf{r})\right]$ is well known to exist, because $v^{\text {ext }}(\mathbf{r})$ determines the many-particle Hamiltonian, Eq. (2) is less obvious. In other words, Hohenberg and Kohn realized that for the ground state the functional $n(\mathbf{r})=n[\Psi]=\left\langle\Psi\left|\sum_{i} \delta\left(\mathbf{r}-\mathbf{r}_{i}\right)\right| \Psi\right\rangle$ can be inverted, i.e., $\Psi=\Psi[n(\mathbf{r})]$. With the help of this theorem the variational problem of the many-particle Schrödinger equation transforms to a variational problem of an energy functional:

$$
E_{0} \leq\langle\Psi|H| \Psi\rangle=E_{v}[\Psi[n]]=E_{v}[n]
$$

Here $E_{0}$ is the energy of the ground state, and $E_{v}[n]=\int d \mathbf{r} v^{\operatorname{ext}}(\mathbf{r}) n(\mathbf{r})+G[n]$. In this functional $n(\mathbf{r})$ is the variable (the electron ground-state density of any $N$-electron system), and $v^{\text {ext }}(\mathbf{r})$ is kept fixed. $G[n]$ is a universal functional independent of the system, i.e., independent of $v^{\operatorname{ext}}(\mathbf{r})$. For example, $G[n]$ is the same for an H-atom, a COmolecule, a solid etc. The main advantage of this approach is that $n(\mathbf{r})$ only depends on three variables while $\Psi\left(\left\{\mathbf{r}_{i}\right\}\right)$ depends on many variables (the $3 N$ coordinates of all electrons). ${ }^{13}$ Thus, it is plausible that the variational problem of $E_{v}[n]$ is easier to solve than that of $\langle\Psi|H| \Psi\rangle$, yet the result for the ground-state energy and the ground state electron density will be the same. The total energy entering Eq. (1) is ${ }^{14}$

$$
E^{\mathrm{tot}}\left(\left\{\mathbf{R}_{J}\right\}\right)=E_{0}\left(\left\{\mathbf{R}_{J}\right\}\right)+\frac{1}{2} \sum_{J, J^{\prime}, J \neq J^{\prime}} \frac{Z_{J} Z_{J^{\prime}}}{\left|\mathbf{R}_{J}-\mathbf{R}_{J^{\prime}}\right|}
$$

where $\left\{\mathbf{R}_{J}\right\}$ includes all atoms, and $Z_{J}$ is the nuclear charge.

An important problem remains, namely that an explicit form of the functional $G[n]$ is unknown. Earlier work (in particular the Thomas-Fermi approach) had shown that the treatment of the kinetic energy $\left\langle\Psi\left|-\frac{1}{2} \nabla^{2}\right| \Psi\right\rangle$ is of particular importance and Kohn and Sham ${ }^{15}$ therefore wrote the energy functional in the form

$$
E_{v}[n]=T_{s}[n]+\int d \mathbf{r} v^{\mathrm{ext}}(\mathbf{r}) n(\mathbf{r})+\frac{1}{2} \int d \mathbf{r} v^{\mathrm{H}}(\mathbf{r}) n(\mathbf{r})+E^{\mathrm{xc}}[n],
$$

where $T_{s}[n]$ is the functional of the kinetic energy of a system of non-interacting electrons with density $n(\mathbf{r})$, and $v^{\mathrm{H}}(\mathbf{r})=\int d \mathbf{r}^{\prime} \frac{n\left(\mathbf{r}^{\prime}\right)}{\left|\mathbf{r}-\mathbf{r}^{\prime}\right|}$ is the Hartree potential that describes the electrostatic interaction between electrons. $E^{\mathrm{xc}}[n]$ is the so-called exchangecorrelation functional. It accounts for the Pauli principle, dynamical correlations due 
to the Coulomb repulsion, and the correction of the self-interaction included for convenience in the Hartree term. With Eq. (5) the problem of the unknown functional $G[n]$ is transformed to one involving $T_{s}[n]$ and $E^{\mathrm{xc}}[n]$. We note in passing that the functional defined by Eq. (5) can be also modified by adding terms which vanish at the correct electron density. Such a functional $E_{v}[n]$ may converge faster towards the ground state or may depend less sensitive on the input density. The latter implies that the input density does not need to be very good, yet the resulting energy represents an acceptable approximation for the correct total energy (see e.g., Ref. 16). Although the functional $T_{s}[n]$ is not known explicitly in a mathematically closed form, it can be evaluated exactly by using the following "detour" proposed by Kohn and Sham. The variational principle applied to Eq. (5) leads to

$$
\begin{aligned}
\frac{\delta E_{v}[n]}{\delta n(\mathbf{r})} & =\frac{\delta T_{s}[n]}{\delta n(\mathbf{r})}+v^{\mathrm{ext}}(\mathbf{r})+v^{\mathrm{H}}(\mathbf{r})+\frac{\delta E^{\mathrm{xc}}[n]}{\delta n(\mathbf{r})} \\
& =\frac{\delta T_{s}[n]}{\delta n(\mathbf{r})}+v^{\mathrm{eff}}(\mathbf{r})=\mu,
\end{aligned}
$$

where $\mu$ is the Lagrange multiplier associated with the requirement of a constant particle number and thus equals the electron chemical potential. The effective potential is defined as

$$
v^{\mathrm{eff}}(\mathbf{r})=v^{\mathrm{ext}}(\mathbf{r})+v^{\mathrm{H}}(\mathbf{r})+v^{\mathrm{xc}}(\mathbf{r}),
$$

with $v^{\mathrm{xc}}(\mathbf{r})=\delta E^{\mathrm{xc}}[n] / \delta n(\mathbf{r})$, and $n(\mathbf{r})$ is a ground-state density of any non-interacting electron system, i.e.,

$$
n(\mathbf{r})=\sum_{i=1} f_{i}\left|\phi_{i}(\mathbf{r})\right|^{2}
$$

where we introduced the occupation numbers $f_{i}$. Because $T_{s}[n]$ is the kinetic energy functional of non-interacting electrons, Eq. (7) (together with Eq. (91)) is solved by

$$
\left[-\frac{1}{2} \nabla^{2}+v^{\mathrm{eff}}(\mathbf{r})\right] \phi_{i}(\mathbf{r})=\epsilon_{i} \phi_{i}(\mathbf{r})
$$

These are the Kohn-Sham equations, that are to be solved self-consistently together with Eqs. (8) and (9). In principle this gives the exact ground-state electron density and total energy of a system of interacting electrons. However, the functional $E^{\mathrm{xc}}[n]$ is still unknown. Some general properties of this functional and values for some special cases are known. Detailed and very accurate understanding exists for systems of constant electron density. The asymptotic behavior at low and high densities is given by expressions derived by Wigner ${ }^{17}$ and Gell-Mann and Brueckner ${ }^{18}$ and for intermediate densities quantum Monte Carlo calculations have been performed by Ceperley and Alder. ${ }^{19}$ This gives the simple curve shown in Fig. 1, and this result for $\epsilon^{\mathrm{xc}}\left(r_{s}\right):=\epsilon_{\mathrm{LDA}}^{\mathrm{xc}}(n)$ is then used in the functional

$$
E_{\mathrm{LDA}}^{\mathrm{xc}}[n]=\int d \mathbf{r} n(\mathbf{r}) \epsilon_{\mathrm{LDA}}^{\mathrm{xc}}(n(\mathbf{r}))
$$

which is the local-density approximation (LDA). ${ }^{15}$ Thus, in the LDA the many-body effects are included such that for a homogeneous electron gas the treatment is exact and for an inhomogeneous system exchange and correlation are treated by assuming that the system can be composed from many small systems with a locally constant density. 


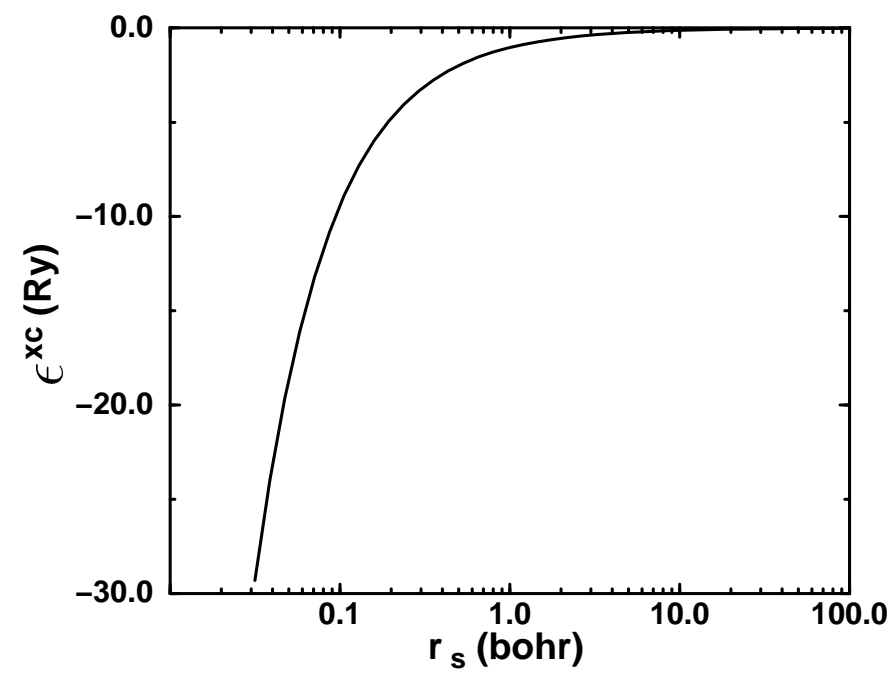

Figure 1. Exchange-correlation energy per particle, $\epsilon^{\mathrm{xc}}$, of homogeneous electron gases with density parameters $r_{\mathrm{s}}$. The electron density and the density parameter are related by $n=\frac{4}{3} \pi r_{s}^{3}$.

The LDA can be improved by including the dependence on the density gradient which leads to the generalized gradient approximation (GGA). Several different GGA's were proposed in the literature ${ }^{20-25}$ and have been used successfully for DFT calculations of atoms, molecules, bulk solids, and surfaces (an overview can be found in Refs. 25 and 26), but also limitations have been identified for example by Mitas et $a l .{ }^{27}$ and Umrigar and coworkers. ${ }^{28}$ It is by now clear that the lattice constants calculated with a GGA are typically larger than those obtained with the LDA, with the experimental values usually being in between. Binding energies (or cohesive energies) of molecules and solids are clearly improved by the GGA and energy barriers of chemical reactions are improved as well (see Ref. 29 and 30 and references therein). Still, for surface diffusion DFT-LDA calculations give energy barriers in good agreement with those deduced from experiments and with GGA calculations. Although the total energies are changed when going from the LDA to the GGA, the changes in energy barriers, i.e., in total energy differences, are typically less pronounced (see e.g., Ref. 31).

\section{IMPLEMENTATION OF DENSITY-FUNCTIONAL THEORY TO COM- PUTE MICROSCOPIC PARAMETERS}

Typically there are only a few candidates for adsorption sites and possible channels for diffusion. This is illustrated in Fig. 2 for the fcc (111) and fcc (100) surfaces. For adatoms which are chemically similar (or equivalent) to those of the substrate the stable sites are those with high coordination and for hopping diffusion the transition state is at the bridge site. The relevant information about the PES then is obtained by calculating the total energy of the system with the adatom placed in those positions. In general, more care is necessary because the bridge site can also be a local minimum of the PES and the energy barrier could be in between the high coordination and the bridge sites. Furthermore, it is possible that the diffusion does not proceed by hopping but by atomic exchange. ${ }^{3-7}$ 
In the bulk crystal the three-dimensional periodicity can be exploited by using Bloch's theorem. Unfortunately, the presence of a surface and an adatom on top of it breaks all periodicity. The (in principle) best approach to treat such difficult situation is given by the Green-function method. ${ }^{32,33} \mathrm{~A}$ popular approximation for (at least in the past) for adsorbate systems is the cluster approach. ${ }^{34}$ The presently most efficient and practical approach that was also used in the results discussed below is the supercell approach. The supercell may be also called a big cluster, but in contrast to conventional cluster calculations the supercell is periodically repeated. As a consequence, the cluster boundary is treated physically very accurately, and by utilizing the periodicity, i.e., the Bloch theorem, it is possible to use very big cells. The idea of an adatom on top of a substrate in the supercell approach is sketched in Fig. 3 . The adatom is placed on top of a slab of a certain number of layers. The number of layers $N_{\text {slab }}$ must be sufficiently large so the adatom does not "feel" the presence of another surface on the other side of the slab (or at least that the quantity to be computed, e.g., a diffusion barrier, is not affected by the other surface). Alternatively, one could also place an adatom on either side of the slab; in this case, there are more symmetries in the geometry but more layers are needed in the slab to screen the mutual interaction between the two adatoms through the slab. The adequate number of layers $N_{\text {slab }}$ depends on the properties that

(111)

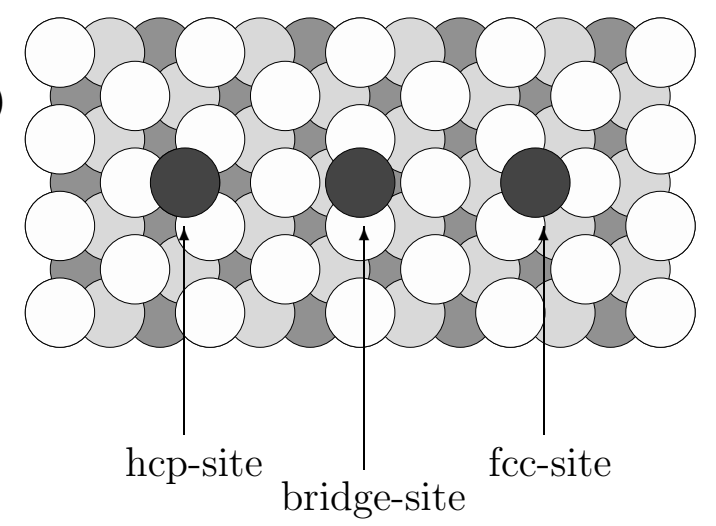

$(100)$

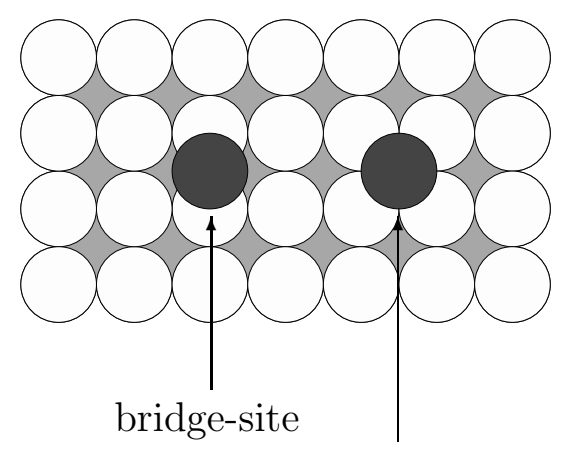

hollow-site

Figure 2. Top view at a fcc (111) and (100) surface. The adsorption sites labeled fcc, hcp, and hollow site usually correspond to the most stable binding sites while the bridge-site is the transition state of a hopping diffusion. 

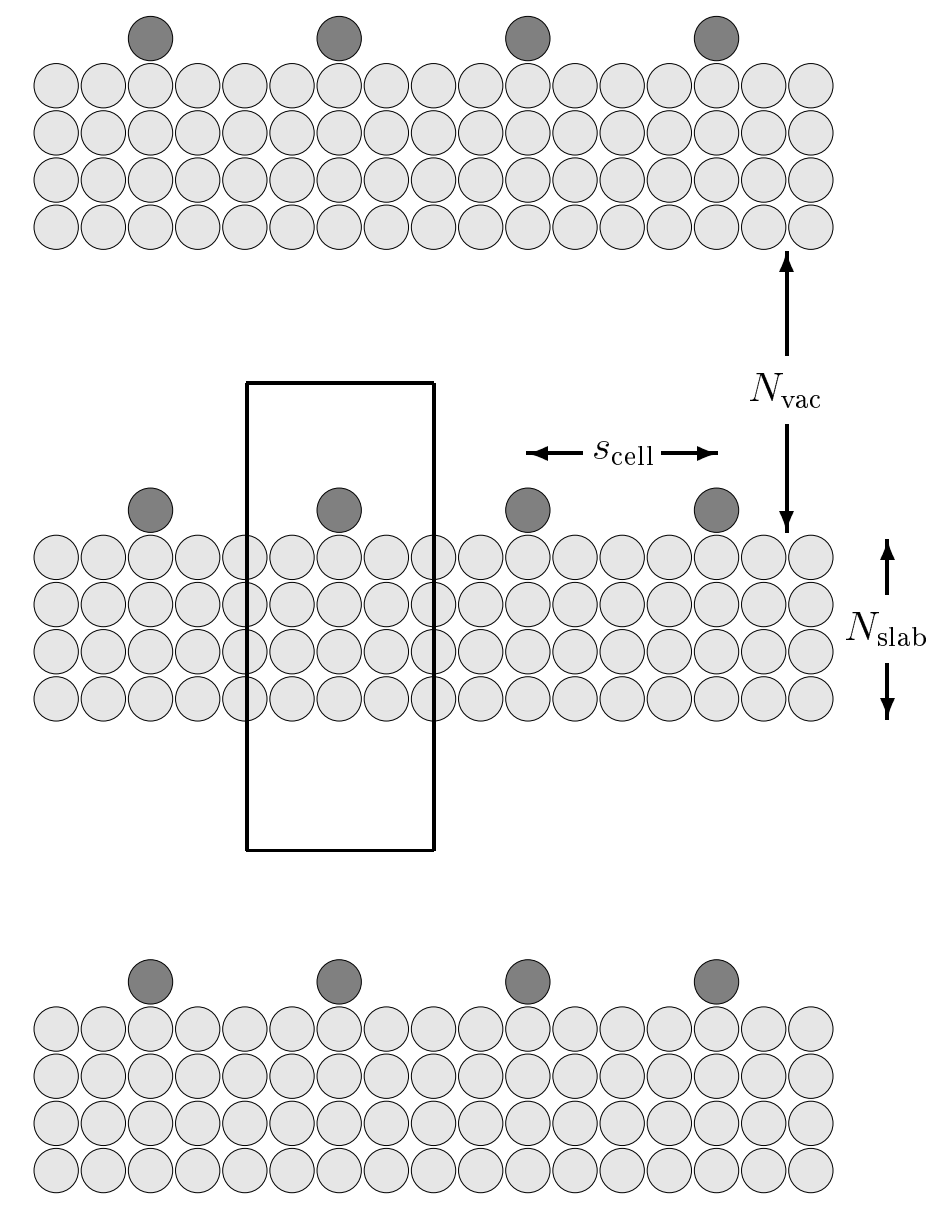

Figure 3. Sketch of a supercell describing an "isolated" adatom at a surface (side view).

one wants to calculate and the surface orientation, and careful tests must be carried out. For example, for the $\mathrm{Ag}$ (111) surface four layers are sufficient when the adatom is placed on only one side of the slab, while for $\mathrm{Al}(100)$ seven layers are necessary.

As illustrated in Fig. 3 the geometry repeats periodically in vertical and lateral directions. The lateral periodicity implies that a single adatom placed on a substrate is not at all a single adatom; if the cell size parallel to the surface is chosen, for example, as $(2 \times 2)$ we actually calculate a system with a coverage of $25 \%$. It is therefore important to test that the interaction with the neighboring adatoms can be neglected. On a (111) surface a cell size of $(2 \times 2)$ is usually sufficient, but sometimes larger cells $[(3 \times 3)$ or even $(4 \times 4)$ ] are necessary. To model a diffusion event along or across a step one either chooses a small island on top of a substrate or a vicinal surface. The latter has the advantage that only one step exists in the unit cell so a smaller cell size is required to attain a negligible step-step interaction. The system also repeats in the vertical direction separated by a vacuum region. The thickness of the vacuum region must be tested as well, but the computational cost of a thicker vacuum region is relatively small compared to a larger cell size or a higher number of slab layers (for a deeper discussion of the above technicalities, see e.g., Ref. 35).

Core electrons typically do not take part directly in the binding process of atoms in molecules and solids, and the nature of the chemical bond is mainly determined by 
the valence electrons. This is exploited by the frozen core approximation where the core electrons are effectively combined with the nuclei to form frozen (i.e. unpolarizable) ions. Still, not just the electrostatic potential but also the quantum nature of the core electrons is felt by the valence electrons. For example, different wave functions must be orthogonal and therefore the valence wave functions have nodes and oscillate in the core region. For practical calculations one needs to expand the wave function in a suitable basis and we choose a plane wave basis $\operatorname{set}^{36}$

$$
\left|\phi_{j}(\mathbf{k}, \mathbf{r})\right\rangle=\sum_{\mathbf{G}} c_{j, \mathbf{k}}(\mathbf{G})|\mathbf{G}+\mathbf{k}\rangle
$$

A plane wave description of wave functions that have nodes and oscillate requires a very large number of plane waves. This inconvenience is cured efficiently by the pseudopotential approach. Modern ab initio pseudopotentials reproduce the potential of an atom exactly outside the core region defined by a radius $r_{c}$ and are rather smooth inside the core region. An important requirement on a "good" pseudopotential is that it is transferable, which means that the pseudopotential should behave like the all-electron potential in a variety of different chemical situations. Pseudopotentials that reproduce the same charge inside the core region as the all-electron potential, and therefore have the same scattering properties, are referred to as norm-conserving. Those that are often used have been developed by Bachelet, Hamann, and Schlüter ${ }^{37}$, Troullier and Martins ${ }^{38}$, and Gonze, Stumpf, and Scheffler. ${ }^{39}$ Recently, Vanderbilt ${ }^{40}$ proposed $a b$ initio pseudopotentials that drop the condition of norm-conservation and therefore can be used with a lower number of plane waves. The gain in computer time due to the smaller basis set is partially compensated by the costs to calculate the correction required by the neglected norm-conservation.

The electron density is calculated according to Eq. (9) as

$$
n(\mathbf{r})=\sum_{\mathbf{k}} \sum_{j} \omega_{\mathbf{k}} f\left(\epsilon_{j}(\mathbf{k})\right)\left|\phi_{j}(\mathbf{k}, \mathbf{r})\right|^{2}
$$

where the integration over the Brillouin zone has been replaced by a sum over a mesh of $\mathbf{k}$-points with $\omega_{\mathbf{k}}$ the $\mathbf{k}$-point's weight. Such replacement typically works quite efficiently because the electron wave functions vary rather smoothly with $\mathbf{k}$, and the main dependence usually is in the phase-factor. Thus, a certain region in $\mathbf{k}$-space is well represented by only one k-point. A convenient scheme to construct an appropriate $\mathbf{k}$-point mesh is described by Monkhorst and Pack. ${ }^{41}$ In ab initio pseudopotential calculations some matrix elements and some integrals are efficiently evaluated in real space whereas others are efficiently evaluated in reciprocal space. The technique of fast Fourier transformation enables a numerically fast change from one representation to the other. Technical details of the computational procedures are described for example in Refs. 8 and 10.

\section{RESULTS FOR MICROSCOPIC PARAMETERS}

We now discuss some results obtained by DFT calculations that provide a deeper insight into the microscopic mechanisms behind growth phenomena. The main objective is to identify the nature and to determine the energetics of diffusion processes. For

the homoepitaxial growth of metallic systems such as $\mathrm{Al} / \mathrm{Al}(111),{ }^{35,42} \mathrm{Al} / \mathrm{Al}(100),{ }^{35,43}$ 
and $\mathrm{Ag} / \mathrm{Ag}(100)^{7,31}$ comprehensive DFT studies have determined diffusion barriers for diffusion on the flat surface and along and across step edges with the hopping and the exchange mechanism. In this section the properties of $\mathrm{Ag} / \mathrm{Ag}$ (111) are discussed and the next section extends earlier DFT calculations of $\mathrm{Al} / \mathrm{Al}(111)^{35,42}$ and analyzes actual growth conditions of mesoscopic islands on long time scales.

Growth of one material on a different material is of particular interest for a number of technological applications. In such a heteroepitaxial system with usually different lattice constants the material to be deposited is under the influence of epitaxial strain. Growth of Ag on Pt (111) and Ag on a thin Ag film on Pt (111) has been the focus of a number of recent studies, ${ }^{44-46}$ and with a lattice mismatch of $4.2 \%$ it provides important information about the effects of strain during growth. Here, we will particularly discuss how strain affects the surface diffusion barrier.

Only few theoretical studies of the influence of lattice mismatch on the diffusion barrier are present in the literature. For a metallic system we are only aware of results for $\mathrm{Ag}$ on $\mathrm{Ag}$ (111) where the authors of Ref. 44 find in a semi-empirical effective medium theory (EMT) calculation that the diffusion barrier increases under tensile strain and decreases under compressive strain.

Here, we present first-principles calculations (more details are given in Ref. 47) studying the dependence of the diffusion barrier on the lattice constant for $\mathrm{Ag}$ on $\mathrm{Ag}(111) .{ }^{44}$ In the range of $\pm 5 \%$ strain the DFT results exhibit an approximately linear dependence with a slope of $\sim 0.7 \mathrm{eV}$ (see Fig. (4). The calculated diffusion barrier for the unstrained system, $E_{\mathrm{d}}^{\mathrm{Ag}-\mathrm{Ag}}=81 \mathrm{meV}$, is in good agreement (within the error margins of the experiment and the calculations) with the scanning tunneling microscopy $(\mathrm{STM})$ results of $E_{\mathrm{d}}^{\mathrm{Ag}-\mathrm{Ag}}=97 \mathrm{meV}$. The accordance between experiment and theory extends to the system $\mathrm{Ag} / \mathrm{Pt}$ (111) and $\mathrm{Ag} / 1 \mathrm{ML} \mathrm{Ag} / \mathrm{Pt}$ (111). These results are summarized in Table 1 . In Fig. The DFT-LDA results are compared to those of an EMT study. ${ }^{44}$ The EMT results exhibit a linear dependence only for very small values of strain $( \pm 2 \%)$ and the diffusion barrier starts to decrease for values of misfit larger than $3 \%$. Indeed, it is plausible that a decrease of the diffusion barrier occurs when the atoms are separated far enough that eventually bonds are broken. However, as our DFT-LDA results show, for Ag/Ag (111) this happens at values for the misfit that are

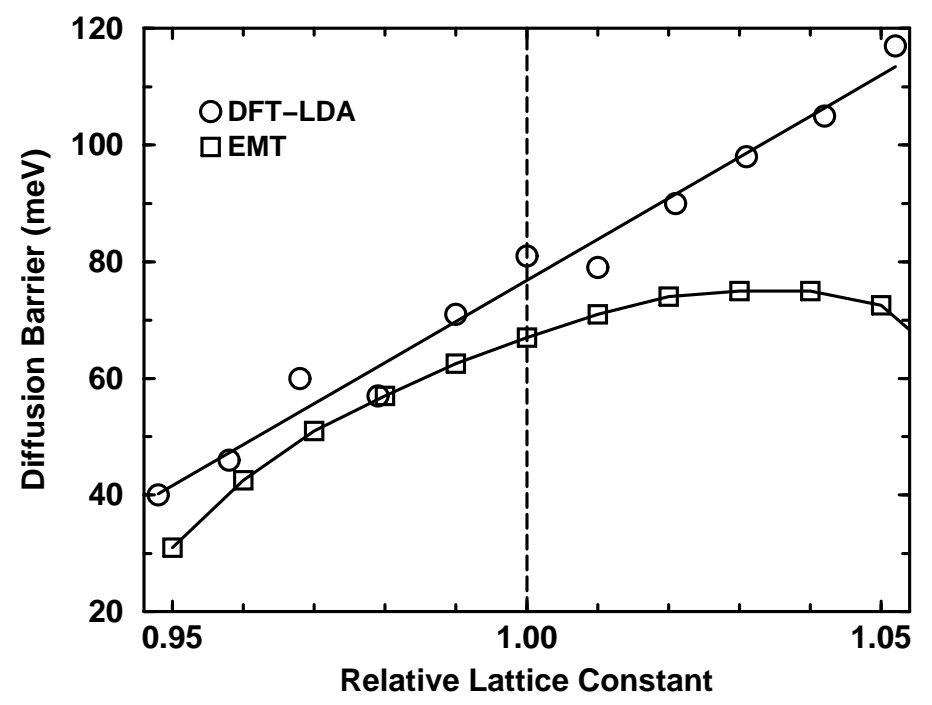

Figure 4. Diffusion barrier (in $\mathrm{meV}$ ) for $\mathrm{Ag}$ on $\mathrm{Ag}(111)$ as function of strain. The circles are DFT-LDA results from Ref. 47 and the squares are EMT results from Ref. 44. 
larger than 5\%. Additionally, when comparison with experiment is possible [i.e., Ag on $\mathrm{Ag}(111)$, and $\mathrm{Ag}$ on a monolayer $\mathrm{Ag}$ on $\mathrm{Pt}(111)]$ the EMT results are off by a factor that varies from 1.2 to 2 .

The DFT results in Fig. 甘were obtained with the LDA for the exchange-correlation functional and test calculations show that GGA increases the diffusion barrier by no more than 5-10\%. This has also been found for Pt on $\mathrm{Pt}(111)$ and $\mathrm{Ag}$ on $\mathrm{Ag}(100)$ (Refs. 48 and 31, respectively). The general trend of an increasing energy barrier for hopping diffusion with increasing lattice constant is quite plausible (for exchange diffusion, see Ref. 7). Smaller lattice constants correspond to a reduced corrugation of the surface, and as a result of a large compression the atom is not bonded much stronger at the adsorption sites than at the bridge site. In contrast, when the surface is stretched the corrugation increases and the adsorption energy at the three-fold coordinated hollow sites increases. This picture will change when the strain is so large that bonds are broken and then it is expected that the hopping diffusion barrier will start to decrease again at very large tensile strain.

It is worth noting that the diffusion barrier for $\mathrm{Ag}$ on top of a pseudomorphic layer of $\mathrm{Ag}$ on $\mathrm{Pt}$ (111) is substantially lower than that for $\mathrm{Ag}$ on $\mathrm{Ag}$ (111). A question that arises is whether this reduced diffusion barrier is a result of the compressive strain or electronic effects due to the Pt substrate. The diffusion barrier for $\mathrm{Ag}$ on $\mathrm{Ag}(111)$ with a lattice constant that is compressed to the value of the lattice constant for $\mathrm{Pt}$ is $E_{\mathrm{d}}^{\mathrm{Ag}-\mathrm{Ag}}=60 \mathrm{meV}$ while that for $\mathrm{Ag}$ on $\mathrm{Pt}(111)$ (also with the Pt lattice constant of $3.92 \AA$ obtained from DFT) is $E_{\mathrm{d}}^{\mathrm{Ag}-\mathrm{Ag} / \mathrm{Pt}}=65 \mathrm{meV}$. The agreement of these two values suggests that the reduction of the diffusion barrier for $\mathrm{Ag}$ on a layer of $\mathrm{Ag}$ on $\mathrm{Pt}(111)$ is mainly a strain effect and that the diffusion barrier on top of a layer of $\mathrm{Ag}$ is essentially independent of the substrate underneath.

We note in passing that the increase of the hopping diffusion barrier with tensile strain is also to be expected (and found) for the more open (100) surface. On the other hand, for exchange diffusion the slope of the energy barrier as a function of strain has the opposite sign and it has been argued ${ }^{7}$ that this behavior and the large surface stress at late $5 d$ transition metals actuate exchange diffusion experimentally found for $\operatorname{Ir}(100)$ and $\mathrm{Pt}(100)$.

\section{$A B$ INITIO KINETIC MONTE CARLO SIMULATIONS}

The time between two successful diffusion events is often of the order of nanoseconds. Since molecular dynamics (MD) calculates all unsuccessful attempts (usually $\sim 10^{3}$ ) explicitly, a typical MD simulation can cover at most times of some picoseconds, possibly some nanoseconds. Therefore, although MD simulations can provide important insight into elementary microscopic mechanisms, they normaly cannot be

\begin{tabular}{|l|c|c|c|}
\hline \hline System & Experiment (Ref. 44) & EMT (Ref. 44) & DFT (Ref. 47) \\
\hline $\mathrm{Ag} / \mathrm{Pt}(111)$ & 157 & 81 & 150 \\
$\mathrm{Ag} / 1 \mathrm{ML} \mathrm{Ag} / \mathrm{Pt}(111)$ & 60 & 50 & 65 \\
$\mathrm{Ag} / \mathrm{Ag}(111)$ & 97 & 67 & 81 \\
\hline
\end{tabular}

Table 1. Diffusion barriers (in meV) for Ag on Pt (111), Ag on one monolayer (ML) $\mathrm{Ag}$ on $\mathrm{Pt}(111)$, and $\mathrm{Ag}$ on $\mathrm{Ag}(111)$. 
used for growth studies. Instead, the method of choice for studying the spatial and temporal development of growth is kinetic Monte Carlo (KMC). The key idea behind $\mathrm{KMC}$ is to describe stochastic processes (such as deposition, diffusion, desorption, etc.) on the microscopic scales by rates and thus to avoid the explicit calculation of unsuccessful attempts. Yet, the result of a KMC study will be the same as that of an MD simulation, provided that the underlying PES is the same. The strategy of KMC can be summarized as follows:

1) Determination of all processes $j$ that possibly could take place with the actual configuration of the system.

2) Calculation of the total rate $R=\sum_{j} \Gamma^{(j)}$, where the sum runs over the possible processes [see step 1)]. Deposition is accounted for in this description by the deposition rate $F$.

3) Choose a random number $\rho$ in the range $(0,1]$.

4) Find the integer number $l$ for which

$$
\sum_{j=1}^{l-1} \Gamma^{(j)} \leq \rho R<\sum_{j=1}^{l} \Gamma^{(j)}
$$

5) Let process $l$ to be taken place.

6) Update the simulation time $t:=t+\Delta t$ with $\Delta t=-\ln (\rho) / R$.

7) Go back to step 1).

KMC simulations have been used to study crystal growth of semiconductors (e.g., Refs. 49-51) and metals (e.g., Refs. 52-55). However, most of these studies have been based on restrictive approximations. For example, the input parameters, such as activation barriers, have been treated as effective parameters determined rather indirectly, e.g. from the fitting of experimental quantities, like intensity oscillations in helium atom scattering (HAS) measurements, in reflection high energy electron diffraction (RHEED) experiments, or island densities from STM pictures. Thus, the connection between these parameters and the microscopic nature of the processes may be somewhat uncertain. Often even the surface structure was treated incorrectly, i.e., the simulation was done on a simple cubic lattice while the system of interest had an fcc or bcc structure. Despite these approximations, such studies have provided significant qualitative and in some cases also quantitative insight into growth phenomena. The next better approach is to use semi-empirical calculations such as the embedded atom method or effective medium theory to evaluate the PES for KMC simulations of growth. ${ }^{56-58}$ The best, but also most elaborate approach to obtain the PES was described in the previous sections. In the following the DFT results for $\mathrm{Al}$ on $\mathrm{Al}$ (111) obtained by Stumpf and Scheffler ${ }^{35,42}$ are utilized for KMC simulations. Thus, it is our aim to perform a realistic simulation that takes into account the correct structure of the system and rate constants determined from accurate $a b$ initio calculations.

On the (111) surface of an fcc crystal there are two different types of close-packed steps, shown in Fig. 5. They are labeled according to their $\{100\}$ and $\{111\}$ facets, referring to the plane passing through the adatom of the step and the neighbor atom 

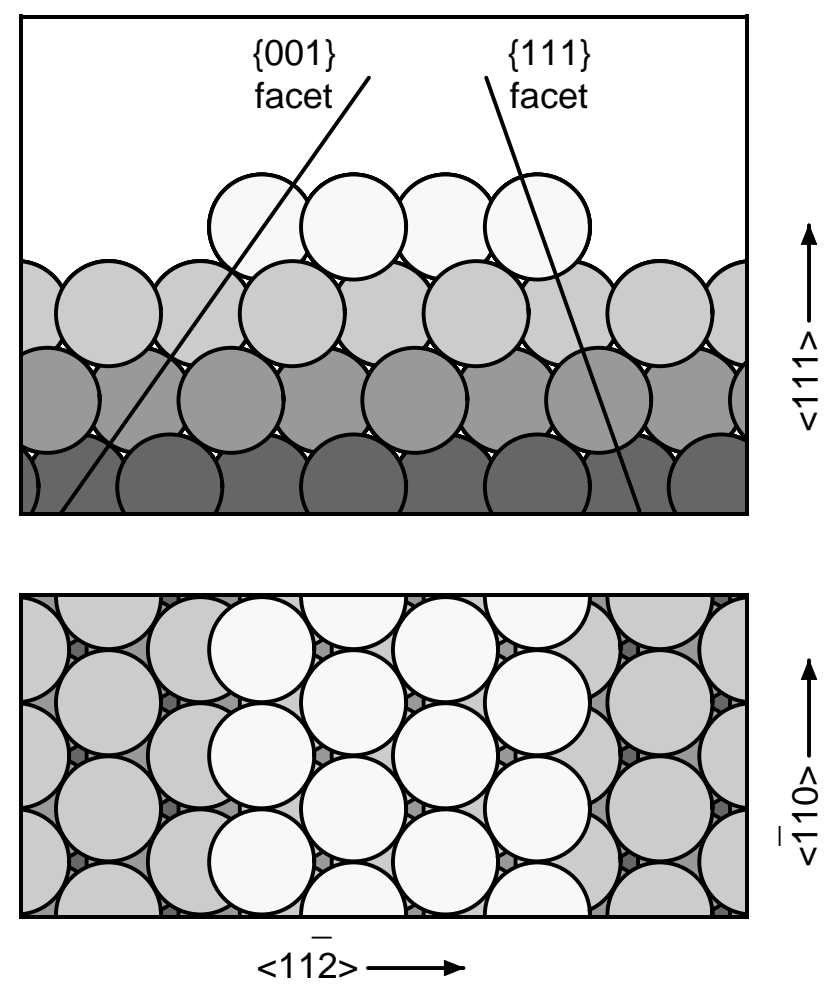

Figure 5. Side view (upper panel) and top view (lower panel) of the two types of close-packed steps on the (111) surface of an fcc crystal.

of the substrate (often these two steps are labeled A and B, respectively). Experimentally it has been shown that for $\mathrm{Pt}(111)^{59}$ and $\operatorname{Ir}(111)^{60}$ these two steps behave quite differently with respect to surface diffusion and growth. For Al(111) the DFT calculations ${ }^{35,42}$ predict that the formation energies of the two steps are different with a lower energy cost for the formation of the $\{111\}$ faceted step than of the $\{100\}$ faceted step: $0.232 \mathrm{eV}$ per atom vs. $0.248 \mathrm{eV}$ per atom. This difference affects the equilibrium shape of the islands as determined by the Wulff construction. Because more open steps have a higher formation energy, one expects in thermodynamic equilibrium and at not too high temperatures hexagonally shaped islands where the edges alternate between those with a $\{100\}$ and a $\{111\}$ microfacet, the latter being longer.

We now like to analyze typical growth conditions, i.e., a situation far from equilibrium where kinetic processes are dominant. For $\mathrm{Al}$ on $\mathrm{Al}$ (111) Stumpf and Scheffler ${ }^{35,42}$ analyzed microscopic diffusion processes and in particular determined the activation energies $E_{\mathrm{d}}$ for:

(i) diffusion of a single adatom on the flat surface: $E_{\mathrm{d}}=0.04 \mathrm{eV}$;

(ii) diffusion from upper to lower terraces which was found to proceed by an exchange with a step-edge atom: $E_{\mathrm{d}}=0.06 \mathrm{eV}$ for the $\{100\}$ step and $E_{\mathrm{d}}=0.08 \mathrm{eV}$ for the $\{111\}$ step;

(iii) diffusion parallel to the $\{100\}$ step via hopping: $E_{\mathrm{d}}=0.32 \mathrm{eV}(0.44 \mathrm{eV}$ for exchange);

(iv) diffusion parallel to the $\{111\}$ step via exchange: $E_{\mathrm{d}}=0.42 \mathrm{eV}(0.48 \mathrm{eV}$ for hopping). 


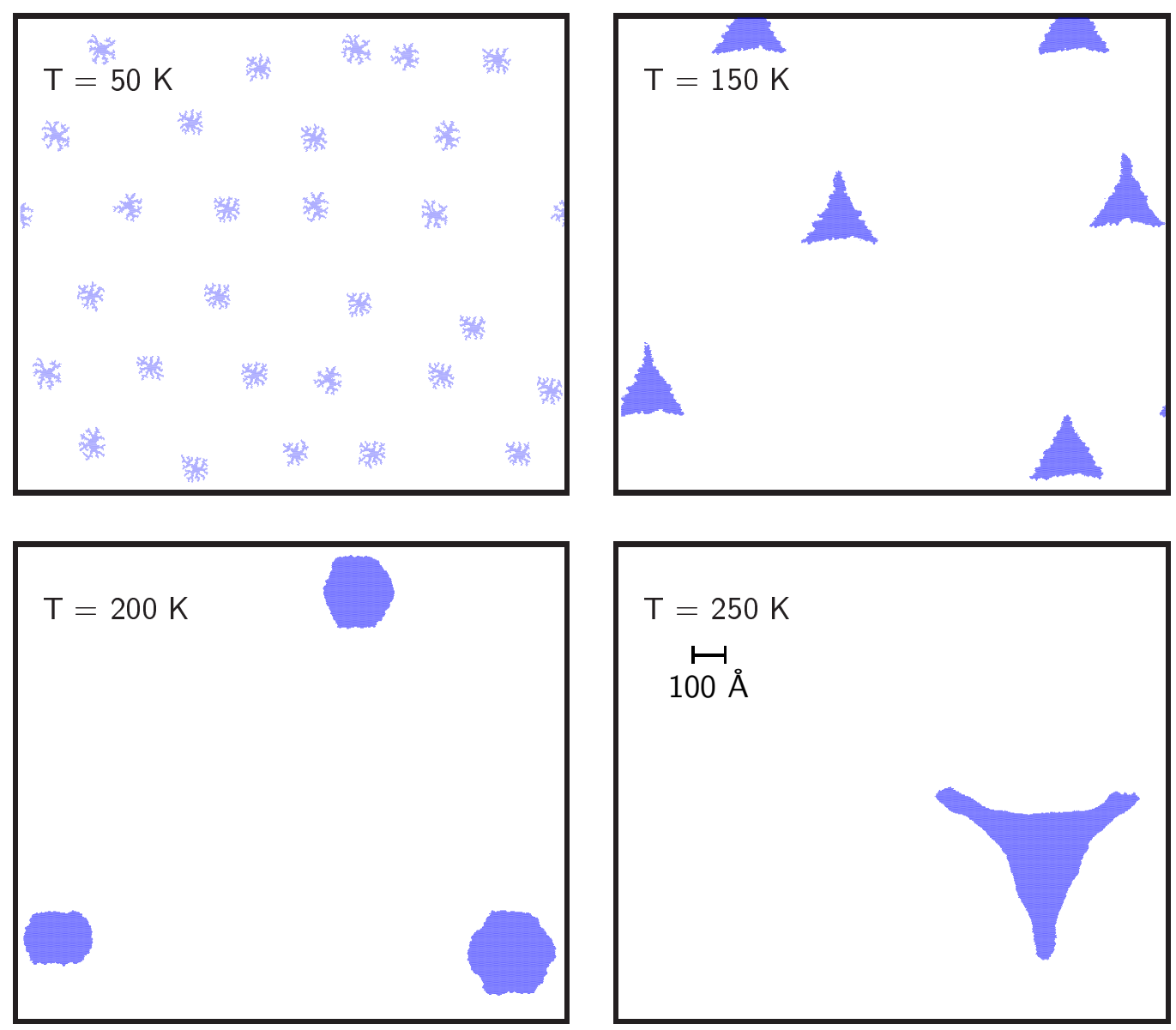

Figure 6. A surface area of $(1718 \times 1488) \AA^{2}$ (half of the simulation array) at four different substrate temperatures. The deposition rate was $0.08 \mathrm{ML} / \mathrm{s}$ and the coverage in each picture is $\Theta=$ 0.04 ML.

The DFT calculations ${ }^{35}$ give that the binding energy of two adatoms in a dimer is $0.58 \mathrm{eV}$, and we therefore assume that dimers, once they are formed, are stable, i.e., they will not dissociate. Moreover, in the lack of reliable information we assume that dimers are immobile. We note that the reported value ${ }^{35,42}$ for the self-diffusion energy barrier is rather low $(0.04 \mathrm{eV})$ and comparable to the energy of optical phonons of $\mathrm{Al}(111)$ $(0.03-0.04 \mathrm{eV}) .{ }^{61}$ Thus, simulations at room temperature may not be reliable because the concept of single jumps between nearest neighbor sites is no more valid. A single optical phonon can furnish enough energy to an adatom for leaving its adsorption site and diffusing on the flat surface. At room temperature the level population of optical phonon is high and the adatoms have practically no saddle point and migrate freely on the flat surface. We therefore limited our study to substrate temperatures $T \leq 250 \mathrm{~K}$.

We adopt periodic boundary conditions, and our rectangular simulation area is compatible with the geometry of an fcc (111) surface. The dimensions of the simulation area are $1718 \times 2976 \AA^{2}$. These dimensions are a critical parameter and it is important to ensure that the simulation area is large enough that artificial correlations of neighboring cells do not affect the formation of growth patterns. The mean free path $\lambda$ of a diffusing adatom before it meets another adatom with possible formation of a nucleation center or is captured by existing islands should be smaller than the linear 
dimension of the simulation array. Since $\lambda$ is proportional to $(D / F)^{1 / 6}$ (Ref. 62), we have that (with $F=0.08 \mathrm{ML} / \mathrm{s}$ ) $\lambda \sim 50 \AA$ for $T=50$ and gets as large as $\sim 10^{3}$ $\AA$ for $T=250 \mathrm{~K}$. We see that our cell is large enough (for the imposed deposition rate) for $T \leq 150 \mathrm{~K}$, whereas at higher temperatures the dimensions of the cell are too small, i.e., for $T>150 K$ the island density is determined by the simulation array rather than the physics. Nevertheless, the island shape is determined by local processes (edge diffusions) and is still meaningful.

In the KMC program two additional insights extracted from the DFT calculations are included: $(i)$ the attractive interaction between steps and single adatoms, and (ii) the fact that diffusion processes take place via different mechanisms (hopping or exchange). Particularly the second point plays an important role in our investigation. In several KMC simulations of epitaxial growth the attempt frequency of the diffusion rate has the same value for all the processes, and this value lies usually in the range of a typical optical phonon vibration or the Debye frequency. However, this assumption may not be right. First, processes with larger activation barriers may have a larger attempt frequency than processes with smaller energy barriers. This is a consequence of the compensation effect described, e.g., in Ref. 63. Moreover, processes as hopping and exchange that involve a different number of particles and different bonding configurations may also be characterized by different attempt frequencies. This has been observed ${ }^{64-67}$ for several systems (Rh, Ir, Pt) and implies that the attempt frequency for exchange diffusion can be larger by up to two orders of magnitude than that for hopping. For Al surfaces calculations with the embedded atom method ${ }^{68}$ showed a difference of prefactors of one order of magnitude.

The results of the ab initio KMC simulations are shown in Fig. 6 for coverages of $\Theta=0.04 \mathrm{ML}$. When the substrate temperature is $50 \mathrm{~K}$ during growth the shape of the islands is highly irregular and indeed fractal. Adatoms which reach a step cannot leave it anymore and they even cannot diffuse along the steps. Thus, at this temperature ramification takes place into random directions, and island formation can be understood in terms of the so-called hit and stick model (see also Ref. 69). At a growth temperature $T=150 \mathrm{~K}$ the island shapes are triangular with their sides being

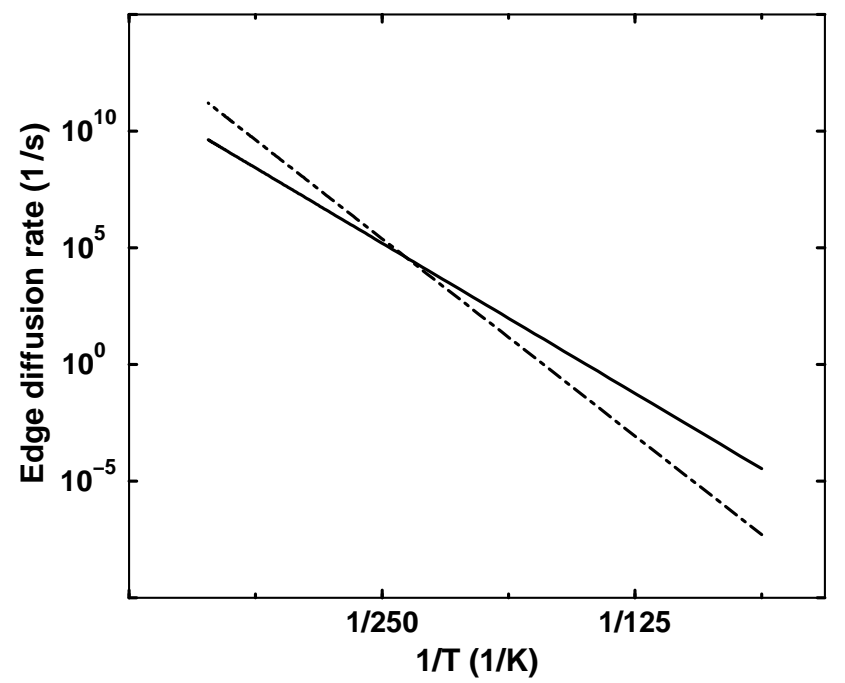

Figure 7. Temperature dependence of the edge diffusion rates for atom diffusion along the $\{100\}$ step by hopping with $\Gamma_{0}=2.5 \times 10^{12} \mathrm{~s}^{-1}$ (solid line), and along the $\{111\}$ step by exchange with $\Gamma_{0}$ $=2.5 \times 10^{14} \mathrm{~s}^{-1}$ (dash-dotted line). 
$\{100\}$ steps. Increasing the temperature to $T=200 \mathrm{~K}$ a transition from triangular to hexagonal shape occurs and for $T=250 \mathrm{~K}$ the islands become triangular again. However, at this temperature they are mainly bounded by $\{111\}$ steps.

To understand the island shapes in the temperature regime between 150 and $250 \mathrm{~K}$, we consider the mobility of the adatoms along the steps (at such temperatures the adatoms at the step edges cannot leave the steps): The lower the migration probability along a given step edge, the higher the step roughness and faster the speed of advancement of this step edge. As a consequence, this step edge shortens as a result of the growth kinetics and eventually it may even disappear. Since diffusion along the densely packed steps on the (111) surface (the $\{100\}$ and $\{111\}$ facets) is faster than along steps with any other orientation this criterion explains the presence of islands which are mainly bounded by $\{100\}$ or $\{111\}$ steps. The same argument can be extended to the diffusion along the two close-packed steps and applied to the triangular islands at $T=150 \mathrm{~K}$, where the energy barrier for the diffusion along the $\{111\}$ facet is larger and thus the $\{100\}$ steps survive so that triangular islands with $\{100\}$ sides are obtained. By considering the energy barriers we would expect only these islands, until the temperature regime for the thermal equilibrium is reached. However, as noted in the introduction, the diffusion of adatoms is not only governed by the energy barrier but also by the effective attempt frequency. For $\mathrm{Al} / \mathrm{Al}(111)$ the effective attempt frequencies have not been calculated, but the analysis of Ref. 35 proposes that the exchange process should have a larger attempt frequency than the hopping process. The results displayed in Fig. 6 are obtained with $1.0 \times 10^{12} \mathrm{~s}^{-1}$ for the diffusion on the flat surface, $2.5 \times 10^{12} \mathrm{~s}^{-1}$ for the jump along the $\{100\}$ step, and $2.5 \times 10^{14} \mathrm{~s}^{-1}$ for the exchange along the $\{111\}$ step. These effective attempt frequencies are the only input of the KMC not calculated explicitly by DFT, but were estimated from the theoretical PES as well as from experimental data for other systems. In Fig. 7 the edge diffusion rates along the two steps are plotted as a function of the reciprocal temperature. At lower temperatures the energy barrier dominates the diffusion rate, but at $T=250 \mathrm{~K}$ the attempt frequencies start to play a role and lead to faster diffusion along the $\{111\}$ facet than along the $\{100\}$ one. Thus, the latter steps disappear and only triangles with $\{111\}$ sides are present. The roughly hexagonally shaped islands at $T=200 \mathrm{~K}$ are a consequence of the equal advancement speed for the two steps at that temperature. Obviously, the temperature-dependence of the growth shapes found in Fig. 6 is crucially determined by the ratio of the two diffusivities and in particular by the temperature at which the two lines of Fig. 7 cross. If the difference were only one order of magnitude, the crossing would be at a temperature that is too high (namely at $T=505 \mathrm{~K}$ ). The formations of fractals (Fig. 6, upper left) and of $\{100\}$ step triangles would still occur. Obviously, the importance of the attempt frequencies should receive a better assessment through accurate calculations, and work in this direction is in progress. While no experimental data for $\mathrm{Al} / \mathrm{Al}(111)$ are presently available we note that a similar sequences of islands as obtained above has been observed for Pt on Pt (111) by Michely et al.. ${ }^{59}$

\section{REFERENCES}

1. P. Ruggerone, C. Ratsch, and M. Scheffler, in The Chemical Physics of Solid Surfaces vol. 8, eds. D.A. King, D.P. Woodruff (Elsevier Science, Amsterdam, 1997), in press.

2. A. Kley and M. Scheffler, in The Physics of Semiconductors, Eds. M. Scheffler and R. Zimmer- 
mann (World Scientific, Singapore, 1996), 1031.

3. D.W. Bassett and P.R. Webber, Surf. Sci. 70, 520 (1978).

4. J.D. Wrigley and G. Ehrlich, Phys. Rev. Lett. 44, 661 (1978).

5. $\quad$ G.L. Kellog and P.J. Feibelman, Phys. Rev. Lett. 64, 3143 (1990).

6. $\quad$ P.J. Feibelman, Phys. Rev. Lett. 65, 729 (1990).

7. B.D. Yu and M. Scheffler, submitted for publication.

8. M.C. Payne, M.P. Teter, D.C. Allan, T.A. Arias, and D.J. Joannopoulos, Rev. Mod. Phys. 64, $1045(1992)$.

9. B. Kohler, S. Wilke, M. Scheffler, R. Kouba, and C. Ambrosch-Draxl, Comput. Phys. Commun. 94, 31-48 (1996).

10. R. Stumpf and M. Scheffler, Comput. Phys. Commun. 79, 447 (1994), M. Bockstedte, A. Kley, J. Neugebauer, and M. Scheffler, to be published.

11. P. Hohenberg and W. Kohn, Phys. Rev. 136, B864 (1964).

12. M. Levy, Proc. Natl. Acad. Sci. (USA) 76, 6062 (1979).

13. We limit our discussion in this paper to non-magnetic systems. However, it is straightforward to generalize DFT and to write the total-energy functional in terms of the electron density and the magnetization density (see for example R.M. Dreizler and E.K.U. Gross, Density Functional Theory (Springer Verlag, Berlin, Heidelberg, New York, 1990)).

14. All equations are noted in Hartree atomic units, i.e., the unit of length is 1 Bohr $=0.5292 \AA$, the unit of energy is 1 Hartree $=27.2116 \mathrm{eV}$, and $\hbar=m=e=1$.

15. W. Kohn and L.J. Sham, Phys. Rev. 140, A1133 (1965).

16. R. Haydock and V. Heine, to appear in Comments in Condensed Matter Physics.

17. E.P. Wigner, Phys. Rev. 46, 1002 (1934).

18. M. Gell-Mann and A.K. Brueckner, Phys. Rev. 106, 364 (1957).

19. D.M. Ceperley and B.J. Alder, Phys. Rev. Lett. 45, 566 (1980).

20. J.P. Perdew and Y. Wang, Phys. Rev. B 45, 13244 (1992); J.P. Perdew, in Electronic Structure of Solids '91, eds. P. Ziesche and H. Eschrig (Akademie Verlag, Berlin, 1991), p. 11; J.P. Perdew, J.A. Chevary, S.H. Vosko, K.A. Jackson, M.R. Pederson, D.J. Singh, and C. Fiolhais, Phys. Rev. $B$ 46, 6671 (1992).

21. J.P. Perdew and Y. Wang, Phys. Rev. B 33, 8800 (1986); J.P. Perdew, Phys. Rev. B 33, 8822 (1986); ibid. 34, 7406(E) (1986).

22. Y. Wang and J.P. Perdew, Phys. Rev. B 43, 8911 (1991); J.P. Perdew, Physica B 172, 1 (1991); J.P. Perdew, K. Burke, Y. Wang, unpublished.

23. A.D. Becke, Phys. Rev. A 38, 3098 (1988); J. Chem. Phys. 98, 3892 (1993).

24. C. Lee, W. Yang, and R.G. Parr, Phys. Rev. B 37, 785 (1988).

25. W. Kohn, A.D. Becke, R.G. Parr, J. Chem. Phys. 100, 12974 (1996).

26. M. Ernzerhof, J.P. Perdew, and K. Burke, in Topics in Current Chemistry 180 (Springer Verlag, Berlin, Heidelberg, New York, 1996), 1.

27. L. Mitas and R.M. Martin, Phys. Rev. Lett. 72, 2438 (1994); J.C. Grossman, L. Mitas, and K. Raghavachari, Phys. Rev. Lett. 75, 3870 (1995).

28. C. Fillipi, X. Gonze, and C.J. Umrigar, to be published in Recent Developments and Applications of Density Functional Theory, ed. J.M. Seminario (Elsevier, Amsterdam, 1996).

29. B. Hammer, M. Scheffler, K.W. Jacobsen, and J.K. Norskov, Phys. Rev. Lett. 73, 1400 (1994).

30. B. Hammer and M. Scheffler, Phys. Rev. Lett. 74, 3487 (1995).

31. B.D. Yu and M. Scheffler, Phys. Rev. Lett. 77, 1095 (1996); B.D. Yu and M. Scheffler, Phys. Rev. B, in press.

32. A.R. Williams, P.J. Feibelman, and N.D. Lang, Phys. Rev. B 26, 5433 (1982); P.J. Feibelman, Phys. Rev. B 35, 2626 (1987).

33. M. Scheffler, C. Droste, A. Fleszar, F. Maca, G. Wachutka, and G. Barzel, Physica 172, 143 (1991); J. Bormet, J. Neugebauer, and M. Scheffler, Phys. Rev. B 49, 17242 (1994).

34. A review on the cluster method is given in: J.L. Witten and H. Yang, Surf. Sci. Rep. 24, 55 (1996).

35. R. Stumpf and M. Scheffler, Phys. Rev. B 53, 4958 (1996).

36. J. Ihm, A. Zunger, and M.L. Cohen, J. Phys. C 12, 4409 (1979).

37. G.B. Bachelet, D.R. Hamann, and M. Schlüter, Phys. Rev. B 26, 4199 (1982); D.R. Hamann, M. Schlüter, and C. Chiang, Phys. Rev. Lett. 43, 1494 (1979); H.S. Greenside and M. Schlüter, Phys. Rev. B 28, 535 (1983); D.R. Hamann, Phys. Rev. B 40, 2980 (1989).

38. N. Troullier and J.L. Martins, Phys. Rev. B 43, 1993 (1991). 
39. X. Gonze, R. Stumpf, and M. Scheffler, Phys. Rev. B 44, 8503 (1991); R. Stumpf, X. Gonze, and M. Scheffler, Research report of the Fritz-Haber-Institut (1990).

40. D. Vanderbilt, Phys. Rev. B 41, 7892 (1990).

41. H.J. Monkhorst and J.D. Pack, Phys. Rev. B 13, 5188 (1976).

42. R. Stumpf and M. Scheffler, Phys. Rev. Lett. 72, 254 (1994); ibid. 73, 508 (1995)(E).

43. P.J. Feibelman, Phys. Rev. Lett. 65, 729 (1990).

44. H. Brune, K. Bromann, H. Röder, K. Kern, J. Jacobsen, P. Stolze, K.W. Jacobsen, J. Nørskov, Phys. Rev. B 52, R14380 (1995).

45. H. Röder, E. Hahn, H. Brune, J.-P. Bucher, and K. Kern, Nature 366, 141 (1993).

46. H. Brune, C. Romalnczyk, H. Röder, and K. Kern, Nature 369, 469 (1994).

47. C. Ratsch, A.P. Seitsonen, and M. Scheffler, Phys. Rev. B 55 (1997).

48. G. Boisvert, L.J. Lewis, and M. Scheffler, unpublished.

49. A. Madhukar, Surf. Sci. 132, 344 (1983); A. Madhukar and S.V. Ghaisas, Appl. Phys. Lett. 47, 247 (1985); S.V. Ghaisas and A. Madhukar, J. Vac. Sci. Technol. B 3, 540 (1985); S.V. Ghaisas and A. Madhukar, Phys. Rev. Lett. 56, 1066 (1986).

50. S. Clarke and D.D. Vvedensky, Phys. Rev. Lett. 58, 2235 (1987); Phys. Rev. B 36, 9312 (1987); Phys. Rev. B 37, 6559 (1988); J. Appl. Phys. 63, 2272 (1988); S. Clarke, M.R. Wilby, D.D. Vvedensky, T. Kawamura, K. Miki, and H. Tokumoto, Phys. Rev. B 41, 10198 (1990); T. Shitara, D.D. Vvedensky, M.R. Wilby, J. Zhang, J.H. Neave, and B.A. Joyce, Phys. Rev. B 46, 6815 (1992); Phys. Rev. B 46, 6825 (1992).

51. H. Metiu, Y.-T. Lu, and Z. Zhang, Science 255, 1088 (1992).

52. M.C. Bartelt and J.W. Evans, Phys. Rev. Lett. 75, 4250 (1995).

53. Z. Zhang, X. Chen, and M. Lagally, Phys. Rev. Lett. 73, 1829 (1994).

54. J.G. Amar and F. Family, Phys. Rev. Lett. 74, 2066 (1995).

55. S.V. Khare, N.C. Bartelt, and T.L. Einstein, Phys. Rev. Lett. 75, 2148 (1995).

56. S. Liu, Z. Zhang, G. Comsa, and H. Metiu, Phys. Rev. Lett. 71, 2967 (1993).

57. J. Jacobsen, K.W. Jacobsen, P. Stolze, and J.K. Nørskov, Phys. Rev. Lett. 74, 2295 (1995).

58. $\quad$ Z.-P. Shi, Z. Zhang, A.K. Swan, and J.F. Wendelken, Phys. Rev. Lett. 76, 4927 (1996).

59. T. Michely, M. Hohage, M. Bott, and G. Comsa, Phys. Rev. Lett. 70, 3943 (1993); T. Michely, private communication; M. Hohage, M. Bott, M. Morgenstern, Z. Zhang, T. Michely, and G. Comsa, Phys. Rev. Lett. 76, 2366 (1996).

60. S.C. Wang and G. Ehrlich, Phys. Rev. Lett. 67, 2509 (1991)

61. J.A. Gaspar and A.G. Eguiluz, Phys. Rev. B 40, 11976 (1989).

62. S. Stoyanov and D. Kashchiev, in Current Topics in Material Science, ed. E. Kaldis (NorthHolland, Amsterdam, 1981), vol. 7, pp. 69 - 141.

63. G. Boisvert, L.J. Lewis, and A. Yelon, Phys. Rev. Lett. 75, 469 (1995).

64. D.W. Bassett and P.R. Webber, Surf. Sci. 70, 520 (1978).

65. G. Ayrault and G. Ehrlich, J. Chem. Phys. 60, 281 (1974).

66. S.C. Wang and G. Ehrlich, Phys. Rev. Lett. 62, 2297 (1989); Surf. Sci. 239, 301 (1990).

67. G.L. Kellog, Surf. Sci. 246, 31 (1991).

68. C.L. Liu, J.M. Cohen, J.B. Adams, and A.F. Voter, Surf. Sci. 253, 334 (1991).

69. T.A. Witten and L.M. Sander, Phys. Rev. Lett. 47, 1400 (1981); Phys. Rev. B 27, 5686 (1983). 